\title{
ARTICLE \\ Neural and neurocognitive markers of vulnerability to gambling disorder: a study of unaffected siblings
}

\author{
Eve H. Limbrick-Oldfield (D) $^{1,2}$, Inge Mick ${ }^{3,4}$, Rachel E. Cocks ${ }^{2,3}$, Remy S. A. Flechais ${ }^{3}$, Samuel Turton (iD ${ }^{3}$, Anne Lingford-Hughes ${ }^{3}$, \\ Henrietta Bowden-Jones ${ }^{5}$ and Luke Clark $\mathbb{D}^{1,2}$
}

\begin{abstract}
Psychological and neurobiological markers in individuals with gambling disorder (GD) could reflect transdiagnostic vulnerability to addiction or neuroadaptive consequences of long-term gambling. Using an endophenotypic approach to identify vulnerability markers, we tested the biological relatives of cases with GD. Male participants seeking treatment for GD $(n=20)$ were compared with a male control group $(n=18)$. Biological siblings of cases with $\mathrm{GD}(n=17$, unrelated to the current GD group) were compared with a separate control group $(n=19)$ that overlapped partially with the GD control group. Participants completed a comprehensive assessment of clinical scales, neurocognitive functioning, and fMRI of unexpected financial reward. The GD group displayed elevated levels of self-report impulsivity and delay discounting, and increased risk-taking on the Cambridge Gamble Task. We did not observe impaired motor impulsivity on the stop-signal task. Siblings of GD showed some overlapping effects; namely, elevated impulsivity (negative urgency) and increased risk-taking on the Cambridge Gamble Task. We did not observe any differences in the neural response to win outcomes, either in the GD or sibling analysis compared with their control group. Within the GD group, activity in the thalamus and caudate correlated negatively with gambling severity. Increased impulsivity and risktaking in GD are present in biological relatives of cases with GD, suggesting these markers may represent pre-existing vulnerability to GD.
\end{abstract}

Neuropsychopharmacology (2020) 45:292-300; https://doi.org/10.1038/s41386-019-0534-1

\section{INTRODUCTION}

Gambling disorder (GD) is a behavioural addiction characterized by a loss of control over gambling to a degree that causes functional impairment, particularly in the financial realm. Research findings in disordered gamblers are sometimes applied to illuminating the 'chicken-and-egg' problem in substance addictions, in which observed differences could reflect either preexisting vulnerability to addiction or the neuroadaptive (or neurotoxic) consequences of chronic substance consumption $[1,2]$. As a 'drug-free addiction', effects in disordered gamblers have been argued to indicate vulnerability uncontaminated by these consequences. Despite its intuitive appeal, this argument is weakened by evidence of neuroplasticity induced by behaviour alone, for example in longitudinal MRI investigations of motor skill learning [3]. The chronic cycle of wins and losses experienced by a problem gambler may cause similar reorganization of brain motivational circuitry.

One way to isolate vulnerability markers is by studying unaffected relatives, in an 'endophenotype' approach [4-6]. Previous studies in first-degree relatives of people with GD display elevated rates of disordered gambling and alcohol use disorders [7-9], but are yet to examine underlying neurocognitive mechanisms. As a high-risk group, biological relatives have both a genetic overlap with the affected individual and may also share environmental risk factors such as childhood adversity [10]. Twin studies indicate that genetic factors contribute around $50 \%$ of the risk of GD $[11,12]$. The current study examines biological siblings of patients with GD, who are expected to express neural and neurocognitive dispositional markers.

We focus on impulsivity, risky decision-making, and reward processing as domains that are central to the pathophysiology of GD. Individuals with GD have been shown to have higher selfreported impulsivity $[13,14]$, choice impulsivity (on delay discounting) [15], and impaired motor impulsivity [16]. Individuals with GD also show elevated risk-taking on decision-making tests including the lowa Gambling Task [17] and Cambridge Gamble Task [18-21]. Similar cognitive alterations are observed in substance use disorders [1]; genetic variation in these traits overlaps with genetic risk for addictive disorders [22], and individual differences have further value in predicting treatment outcomes [23]. Several studies have examined the neural response to rewarding outcomes (i.e. monetary wins) in GD using functional MRI (fMRI), focussing on ventral striatum, medial and orbital prefrontal cortex, and insula. A recent meta-analysis [24] of these studies described reduced activity in this network in individuals with GD [25-27], although other studies have reported no alteration in win reactivity $[28,29]$ or even win-related hyperactivity in GD [30, 31].

This evidence for both hypo- and hyper-reactivity of reward circuitry appeals to distinct theoretical frameworks for addiction,

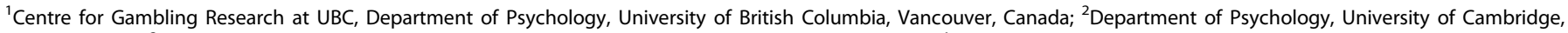

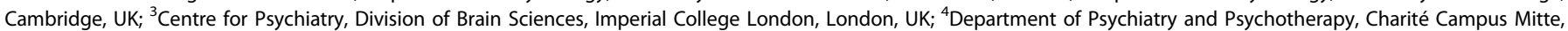
Universitätsmedizin Berlin, Berlin, Germany and ${ }^{5}$ National Problem Gambling Clinic, CNWL NHS Foundation Trust, Imperial College London, London, UK

Correspondence: Eve H. Limbrick-Oldfield (eve@psych.ubc.ca) or Luke Clark (luke.clark@psych.ubc.ca)

Received: 27 March 2019 Revised: 26 August 2019 Accepted: 12 September 2019

Published online: 9 October 2019 
which are relevant to vulnerability. Evidence for reward hypoactivity is interpreted within the reward deficiency framework, which posits that risky, high stimulation behaviours like gambling compensate for a developmentally sluggish reward system $[32,33]$. Conversely, evidence for reward hyper-activity may be interpreted within the incentive salience (or incentive sensitization) hypothesis, that the response to drug- (or gambling-) related stimuli becomes amplified with ongoing use [34, 35]. These two theories are not mutually exclusive: reward deficiency is a theory of addiction vulnerability, whereas incentive salience is a theory of addiction development. For our participants with GD, we hypothesized that impulsivity and risk-taking would be elevated relative to controls. We predicted dysregulation of reward circuitry using our neuroimaging probe, although we did not have a strong directional prediction given inconsistencies in past research [36] and the contrasting effects of reward deficiency and incentive salience. For our high-risk group of unaffected siblings, we predicted elevated impulsivity and risk-taking, and hypo-activity of brain reward circuitry, as neurocognitive and neural expressions specifically aligned with reward deficiency as a vulnerability process. Recent evidence points to such effects for substance use vulnerability, in a two year prospective study of mid-adolescents [37] (see also [38, 39]) as well as in unaffected siblings of stimulant users $[5,40]$, and the present study presents a comparable analysis for GD.

\section{MATERIALS AND METHODS}

Participants

Participants were considered for inclusion if they were aged 18 to 60, had good English comprehension, and no MRI contraindications. Participants were excluded if they suffered from other past or current DSM-IV Axis I psychiatric illnesses with the exception of past depression or anxiety disorders in the GD group, if they were currently taking any psychotropic medications, including over-the-counter opiates, or if they tested positive for illegal drug use using a urine screen or alcohol intoxication using a breath alcohol test, administered on test days (see Supplemental Information). Males with GD $(n=20)$ were recruited from the National Problem Gambling Clinic (NCPG), London, U.K. Diagnosis was confirmed using DSM-IV criteria and corroborated by scores $\geq 8$ on the Problem Gambling Severity Index (PGSI) [41]. An independent group of participants who did not have current or past GD, but whose siblings had current GD $(n=17)$, were recruited through advertisements at the gambling clinic, local support groups for affected others, and local newspapers. A diagnosis of GD in the affected sibling was confirmed by a score $\geq 8$ on the PGSI conducted by telephone with the affected individual. Given the unbalanced gender ratios in the GD (all male) and sibling ( 8 male, 9 female) groups, we recruited a total of 28 control participants (18 male) who were split into two partiallyoverlapping subgroups that were each demographically comparable to the groups of interest. The control group matched to the GD group (conGD) consisted of 18 male participants. The control group matched to the sibling group (conSIB) consisted of nine of these male participants and ten female participants $(n=19)$. The UK National Research Ethics Service approved the protocol, and all volunteers provided written informed consent.

\section{Procedure}

Demographic and clinical characteristics. IQ was assessed using the vocabulary and matrix reasoning subtests of the Wechsler Abbreviated Scale of Intelligence [42]. Participants completed the Beck Depression Inventory (BDI-II) [43], Beck Anxiety Inventory (BAI) [44], Alcohol Use Disorders Identification Test (AUDIT) [45], Drug Abuse Screening Test (DAST) [46], and the Fagerstrom Test for Nicotine Dependence (FTND) [47]. To control for childhood adversity as an environmental risk factor for GD [10, 48], we administered the Childhood Trauma Questionnaire (CTQ) [49]. Forms of gambling were assessed using a modified version of the South Oaks Gambling Screen (SOGS) [50].

Impulsivity and risky decision making. Self-report impulsivity was measured with the UPPS-P Impulsive Behaviour Scale [51]. Participants completed the Monetary Choice Questionnaire (MCQ) [52] as a 27-item measure of delay discounting that derives the hyperbolic discounting parameter, $k$, for three levels of reward magnitude. Participants completed two computerized neurocognitive tasks from the CANTAB assessment (Cambridge Cognition Ltd, Cambridge, UK): the Stop Signal Task [53] as a measure of motor impulsivity, and the Cambridge Gamble Task [54] as a measure of decision-making under risk. For full details on these measures, see Supplemental Information.

fMRI reward task. During their scan, participants completed a slot machine task [29, 55-57] (see Fig. 1). On each trial, the participant selected a play icon on the left reel, and then the right reel spun. If the two icons aligned on the payline, the participant won $£ 0.50$. Other outcomes won nothing. On a third of the trials participants were asked 'How do you rate your chance of winning?' after icon selection and 'How much do you want to continue to play' after outcome. Each participant completed two EPI runs of 42 trials each on a 3T MRI scanner (see Supplemental Information).

\section{Data analysis}

The GD group and the sibling group were compared with their respective control groups in parallel sets of models. In cases where statistical assumptions were violated, we applied the same test in both sets of models. Behavioural analyses were run in $\mathrm{R}$ ( $\mathrm{R}$ Core Team, Vienna); multilevel models were run in $\mathrm{R}$ using Ime4. Analysis scripts are available online (https://github. com/CGR-UBC/gambling-sibling-study).

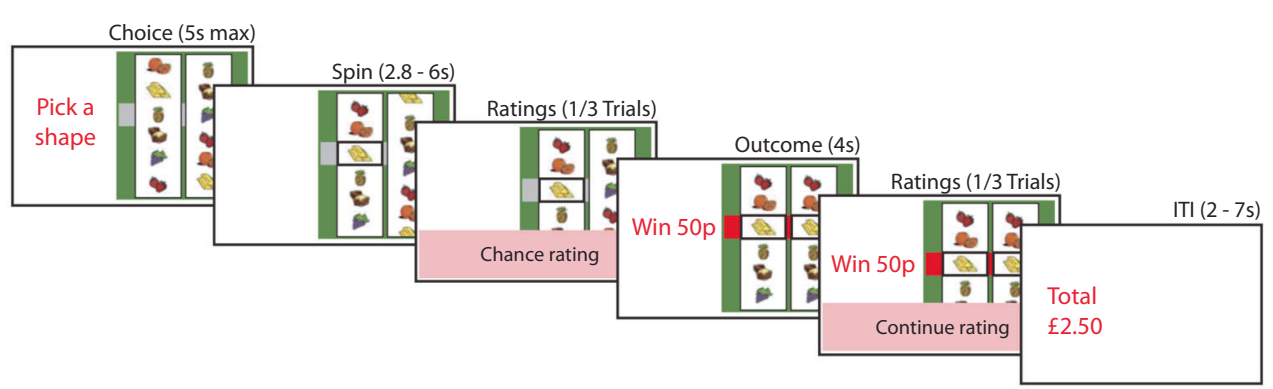

Fig. 1 A win trial on the slot machine task. On miss trials, the icons did not match on the pay line and the words "No win" were shown at outcome. On one third of the trials participants had to provide ratings on for the question "How do you rate your chance of winning?" before the spin, and a "How much do you want to continue to play?" rating after the outcome 
Impulsivity and risky decision making. UPPS-P subscales were compared between groups using Welch's t-tests (two-tailed) which does not assume equal variances. For the MCQ and Cambridge Gamble Task, groups were compared using multilevel regression models (see Supplemental Information). On the Cambridge Gamble Task, two GD and one conGD did not complete the task. For the Stop Signal Task, groups were compared using Wilcoxon rank sum tests; two GD and one conGD did not complete the task, and the staircase algorithm failed to converge in two GD, one sibling, one conGD, and one conSIB.

Functional MRI. Three participants (one GD, one sibling, one control) were fully excluded, and three participants (one GD, two controls) contributed only one run, due to excess in-scanner motion (see Supplemental Information). Two participants (one GD, one sibling) were excluded due to computer error during acquisition. Data are reported from $18 \mathrm{GD}$ compared to 17 conGD, and 15 siblings ( 7 male) compared to 18 conSIB ( 8 male).

Brain imaging data were analyzed using the FMRIB Software Library (FSL). Statistical analyses were carried out in FEAT (FSL Expert Analysis Tool) using a general linear model approach (see Supplemental Information). Outcomes were modelled with four separate regressors; wins, near misses before the payline, near misses after the payline, and full misses $[29,55,56]$. Two firstlevel contrasts were constructed: the first compared win outcomes to all non-win outcomes, the second compared near-miss outcomes to full-miss outcomes. In addition to testing group differences, we tested correlations with gambling severity (PGSI) score within the GD group.

The voxel-wise group-level statistics were masked using the win minus non-win contrast from an independent dataset of 16 healthy participants who completed the same slot machine task with an identical analysis pipeline (Limbrick-Oldfield \& Clark, unpublished; see Supplemental Information) (see Fig. 3a). Foci were family-wise error (FWE) corrected using cluster-based thresholding $(Z=3.1 p<0.05)$. This functionally derived map of reward-related activity was also used to derive three a priori regions of interest proximal to ventral striatum, medial PFC, and anterior insula (see Fig. 3c), which were sensitive to win and nearmiss outcomes in previous experiments with this task $[29,55,56]$. Featquery was used to extract the median percent signal change within each region. All statistical maps reported here are available online (https://neurovault.org/collections/5527/).

\section{RESULTS}

Within the GD group, the median PGSI score was 18 (range 10-25). All other participants scored zero on the PGSI, with the exception of a single sibling scoring one. All GD participants reported engaging in multiple forms of gambling. The GD and conGD groups did not significantly differ in age, WAIS-estimated IQ, childhood trauma (CTQ), or alcohol consumption (AUDIT) (see Table 1a). The GD group reported significantly higher depression (BDI) and anxiety (BAl) scores. The sibling group did not differ significantly from the conSIB group on any demographic or clinical scales, although they did show a non-significant increase in BDI $(r=0.25)$. The groups were also comparable for past 12 month reported smoking and illicit drug use, and within those that did smoke or report drug use, there were no differences in FTND or DAST severity. These group comparisons were repeated for the subsets of participants included in each reported analyses, and the results remained qualitatively unchanged.

Impulsivity and risky decision making

The GD group scored significantly higher than conGD on the Negative Urgency, Positive Urgency, and Lack of Planning subscales of the UPPS-P (Table 1b). The siblings scored significantly higher than conSIB on Negative Urgency.
On delay discounting (MCQ), the GD group showed steeper discounting rates (i.e. elevated impulsivity) than conGD (main effect of group, $b=-0.51,95 \% \mathrm{Cl}[-1.00,-0.023], p<0.05$ ) (Table 1c). There was a significant main effect of reward magnitude $(b=-0.43,95 \% \mathrm{Cl}[-0.54,-0.32], p<0.001)$ that did not differ by group $(b=-0048,95 \% \mathrm{Cl}[-0.16,0.063], p=0.40)$. For the siblings comparison, we observed no overall group difference $(b=0.14,95 \% \mathrm{Cl}[-0.39,0.67], p=0.59)$, and the significant effect of magnitude $(b=-0.53,95 \% \mathrm{Cl}[-0.68,-0.39]$, $p<0.001)$ did not differ between groups $(b=-0.11,95 \% \mathrm{Cl}$ $[-0.26,0.032], p=0.13)$.

For the Stop Signal Task, there were no significant group differences in SSRT, go reaction time, go discrimination errors, or the probability of a successful stop, (Table 1d).

For the Cambridge Gamble Task, we first analyzed decision quality in the GD and conGD groups (Fig. 2a). The addition of box ratio (i.e. the level of risk) significantly improved the model $\left(x^{2}(3)\right.$ $=43.02, p<0.001)$ as did the addition of group $\left(x^{2}(1)=6.30, p<\right.$ $0.05)$, but not the group by box ratio interaction $\left(x^{2}(1)=0.54, p<\right.$ 0.46). Across all participants, as box ratio decreased, participants were less likely to choose the majority colour $(b=-0.77,95 \% \mathrm{Cl}$ $[-1.42,-0.22], p<0.01)$. The GD group chose the majority colour less often than conGD $(b=-2.02,95 \% \mathrm{Cl}[-3.88,-0.47], p<0.05)$. For the siblings analysis (Fig. 2b), the addition of box ratio significantly improved the model $\left(x^{2}(3)=24.56, p<0.001\right.$; box ratio across all participants $b=-1.42,95 \% \mathrm{Cl}[-3.18,-0.10], p<$ $0.05)$, but the addition of group $\left(x^{2}(1)=0.14, p=0.71\right)$ and the group by box ratio interaction did not $\left(x^{2}(1)=3.65, p=0.056\right)$.

In the GD comparisons of bet size (Fig. 2c, e), the addition of box ratio improved the model for both ascending $\left(x^{2}(3)=521.80\right.$ $p<0.0001)$ and descending $\left(x^{2} \quad(3)=429.43 p<0.0001\right)$ bet conditions. As box ratio decreased, the percentage bet decreased (ascending $b=-13.90,95 \% \mathrm{Cl}[-16.37,-11.42], p<0.0001$, descending $b=-12.12,95 \% \mathrm{Cl}[-15.49,-9.08], p<0.0001)$. In the ascending condition, the addition of group significantly improved the model $\left(x^{2}(1)=5.51, p<.05\right)$ such that GD placed higher bets than conGD across all box ratios $(b=-12.39,95 \% \mathrm{Cl}$ $[-16.37,-11.42], p<0.05)$; the group and box ratio interaction did not improve the model $\left(X^{2}(1)=0.51, p=0.47\right)$. In the descending condition, group $\left(x^{2}(1)=0.45, p=0.50\right)$ and the group by box ratio interaction $\left(x^{2}(1)=0.15, p=0.70\right)$ did not improve the model.

In the siblings comparisons of betting (Fig. $2 \mathrm{~d}, \mathrm{f}$ ), we again observed effects of box ratio (ascending: $x^{2}(3)=676.05, p<$ $0.0001, b=-11.74,95 \% \mathrm{Cl}[-13.82,-9.65], p<0.0001$; descending: $X^{2}(3)=440.27, p<0.0001, b=-11.90,95 \%$ Cl $[-14.05$, $-9.72], p<0.01)$. In the ascending condition, the addition of group significantly improved the model $\left(X^{2}(1)=10.68, p<0.01\right)$ such that siblings placed higher bets than conSIB $(b=-17.86,95 \% \mathrm{Cl}$ $[-28.06,-7.66], p<0.01)$, but the group by box ratio interaction did not improve the model $\left(x^{2}(1)=0.029, p=0.87\right)$. In the descending condition, neither group $\left(x^{2}(1)=1.10, p=0.29\right)$ nor the group by box ratio interaction $\left(x^{2}(1)=0.24, p=0.62\right)$ significantly improved the model.

\section{fMRI reward task}

The contrast of win >non-win outcomes revealed significant clusters of activity in all groups (Fig. 3b), including cingulate cortex and frontal pole (see Supplemental Table 2). All groups, with the exception of the sibling group, showed active clusters covering the ventral striatum and orbitofrontal cortex. With cluster-based thresholding, there were no significant differences in activity in either the GD versus conGD comparison, or the sibling versus conSIB comparison. Reward-related circuitry was interrogated further using three ROls - bilateral caudate, paracingulate cortex, and bilateral orbito-frontal cortex - from the independent win mask (Fig. 3a). Both the GD and sibling groups displayed significant win-related signal increases in each of the three ROls 


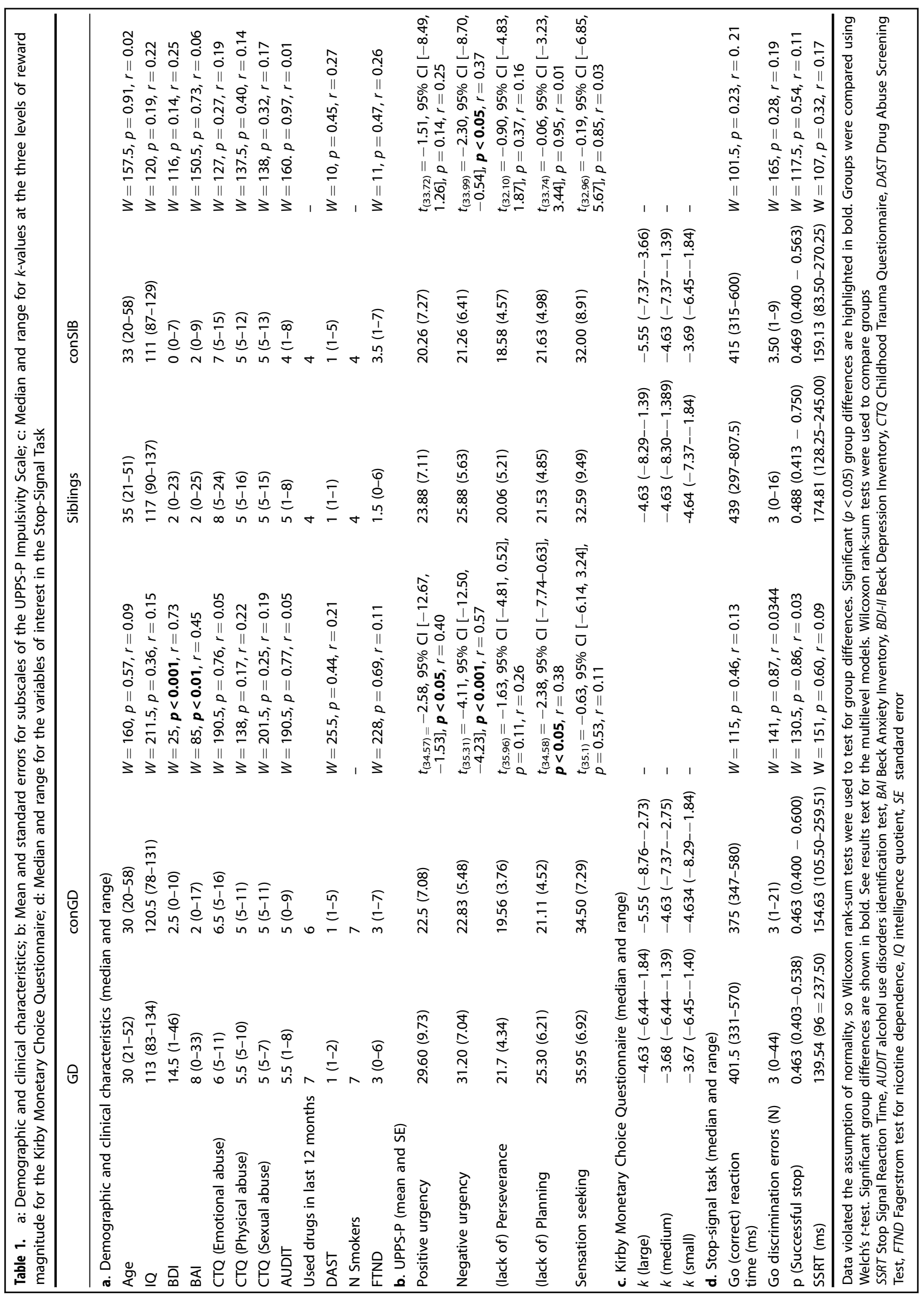



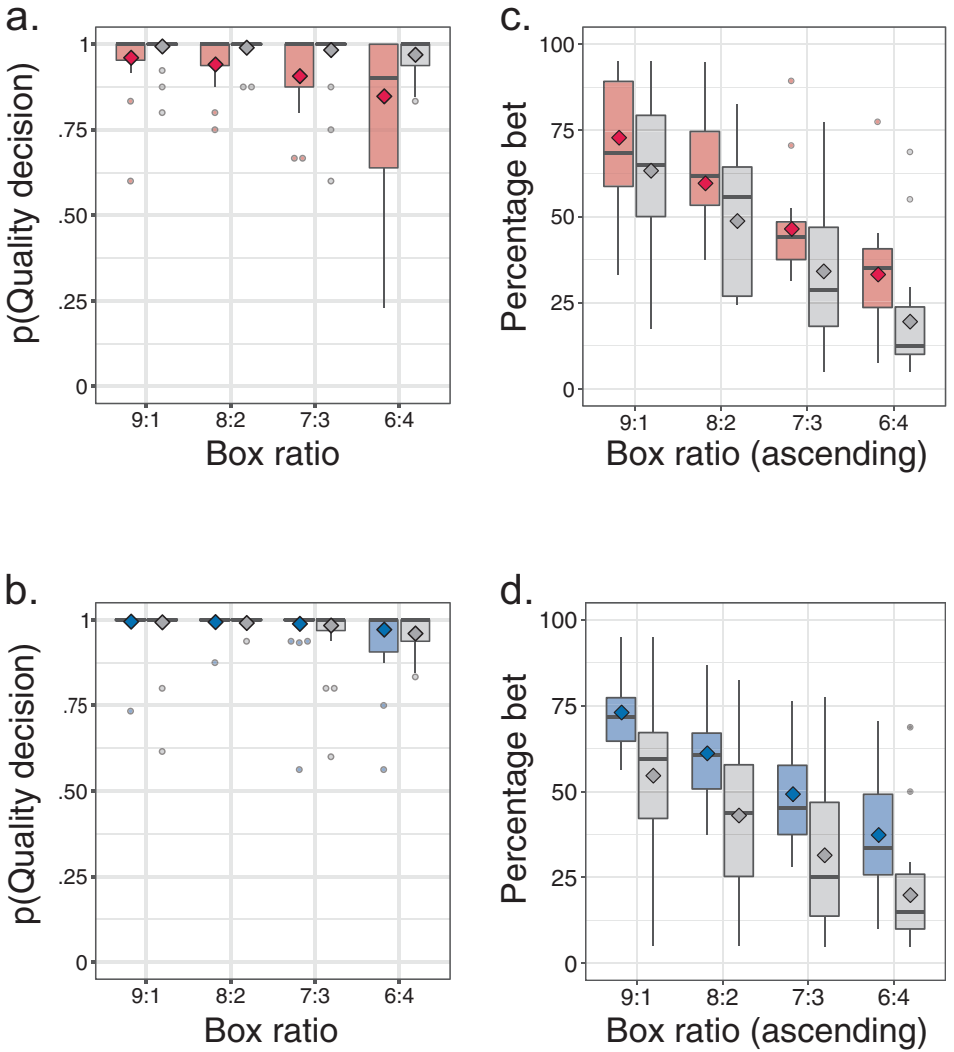

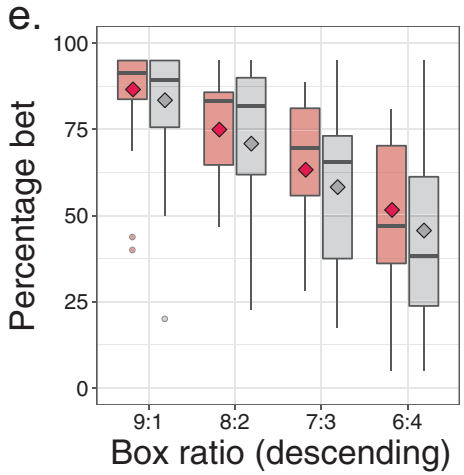

GD ConGD f.

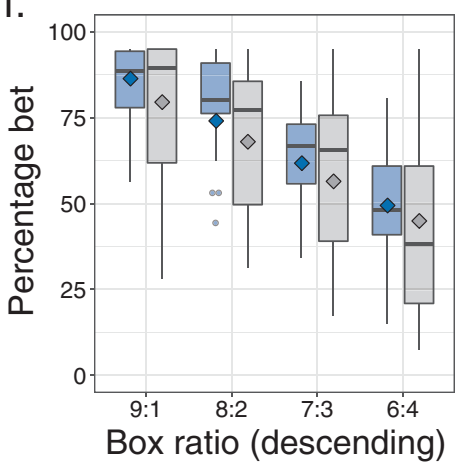

Siblings

Fig. 2 Decision quality and the bet proportion during the Cambridge Gamble Task. Diamonds represent model prediction, observed data shown using Tukey Boxplots. a, b Decision quality as a function of box ratio and group, $\mathbf{c}, \mathbf{d}$ Bet proportion in the ascending condition, $\mathbf{e}, \mathbf{f}$ Bet proportion in the descending condition. Red = Gambling Disorder (GD). Blue = siblings

(Fig. 3c), which did not differ against controls in either set of comparisons.

Within the GD group, win-related activity (win > non-win contrast) was negatively correlated with gambling severity (PGSI) in a single cluster extending from lateral thalamus to bilateral caudate, ( $Z \max =3.86, \mathrm{MNI}=[-16,-2,14], 475$ voxels) (Fig. 4). We did not observe any group differences in near-miss related activity (see Supplemental Information), or on the ratings of 'chances of winning' or post-outcome 'continue to play' (see Supplemental Information).

\section{DISCUSSION}

We show a profile of increased self-reported impulsivity (UPPS$P$ ) and delay discounting (MCQ) in men with GD, in line with previous findings [13-15]. We found strong evidence for elevated negative urgency, with weaker evidence for increases in positive urgency and (lack of) planning subscales, and delay discounting. We found strong evidence of impaired decision making on the Cambridge Gamble Task in individuals with GD, placing both higher bets (a direct marker of risky choice) and also choosing the advantageous box colour less often than controls. From our psychological assessment, there was no evidence of differences on the UPPS-P sensation-seeking subscale (see also [14]), or stop-signal response inhibition, which in prior work can be disrupted in more severe GD cases $[14,16]$. Some of the differences we observed were also detected in biological siblings of GD. The siblings showed increased scores on negative urgency (UPPS-P), although this was a weaker relationship than observed in our GD analysis. The siblings placed significantly higher bets on the Cambridge
Gamble Task, indicative of risk-taking. The difference we observed was greater in the sibling group, compared to the GD analysis (a difference from controls of $\sim 17 \%$ compared to $\sim 12 \%)$. The relative strength of the evidence we observed suggests the group differences we observed on the CGT may hold the most practical relevance as a marker for vulnerability to GD. On other GD-sensitive measures, no significant differences were observed in the siblings, including UPPS-P positive urgency and (lack of) planning subscales, delay discounting, and Cambridge Gamble Task decision quality.

Neuroimaging markers of reward processing were utilized to test reward deficiency as a biomarker of addiction vulnerability. Our hypothesis of reward hypo-activation in the siblings was not supported; indeed, we did not find evidence for any neuroimaging markers of GD vulnerability. As strengths of our analysis, we paid careful attention to in-scanner movement, which can be increased in GD [58], and our analysis focused on the win-related network identified using the same slot machine task in an independent healthy group. Our analysis corroborated win-related activity in the GD and siblings groups, in established reward-sensitive subcortical (striatum) and cortical (medial PFC) regions, consistent with earlier studies using this task $[29,55,56,59]$. There was no evidence for between-group differences in reward signalling in either comparison, although we did observe a negative correlation between win-related activity in striatum and GD severity. Individual differences within GD groups are, in fact, often reported and may reflect the combination of individual differences in vulnerability loading and experience-dependent neuroadaptations [60]. A negative correlation with GD severity can be reconciled more readily with reward deficiency (a greater level of vulnerability associated with reward hypo-activation) than with 
a. Win-mask

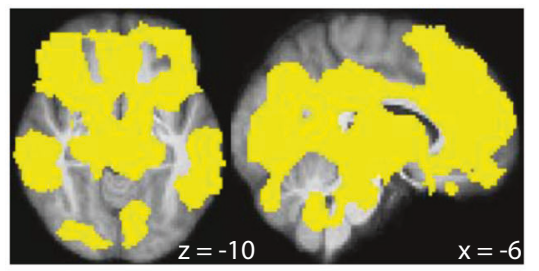

b. Win-mask results

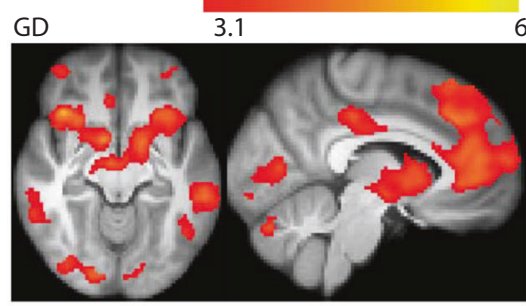

conGD

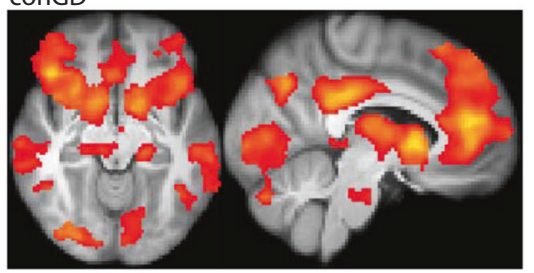

Siblings

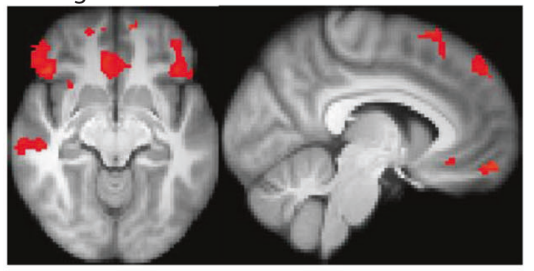

conSIB

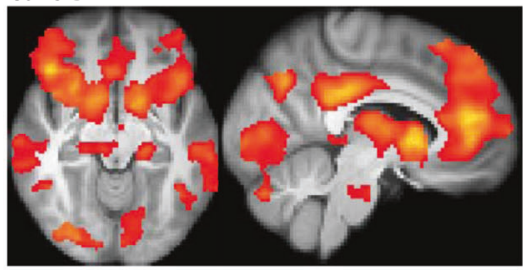

c. ROI results

Caudate
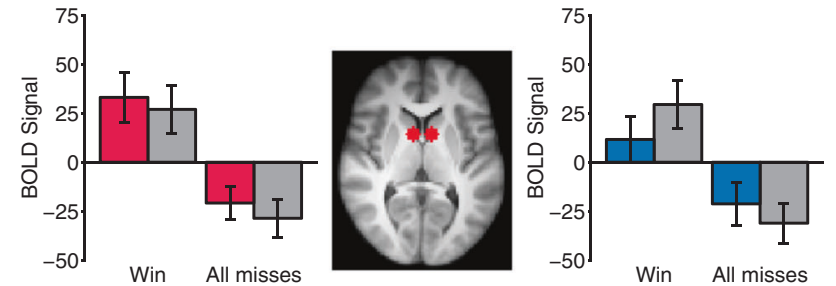

Win All misses

Paracingulate cortex
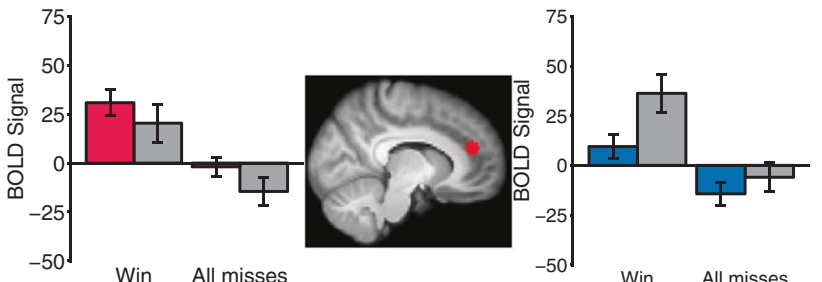

Win All misses

Orbito-frontal cortex

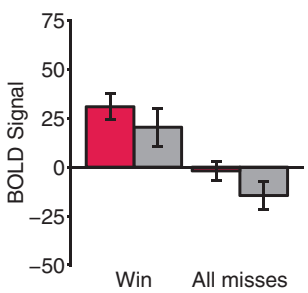

GD

ConGD
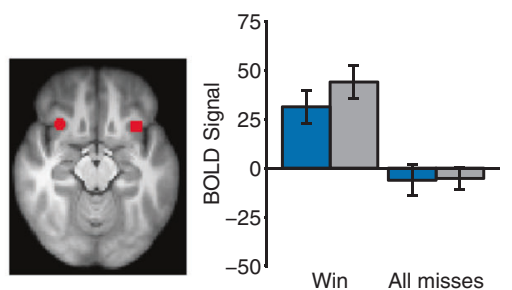

Siblings ConSIB

Fig. 3 Win related fMRI activity derived from the win > non-win contrast in the slot machine task. a Win-mask derived from an independent group of participants. b Significant clusters of activity revealed from our win-mask analysis. See Supplementary table S2 for a list of the peak coordinates of the significant clusters. All images cluster corrected, $Z>3.1, p<0.05$ and presented using radiological convention. c Region-ofinterest analysis of win-related activity in the slot machine tasks. Center panel indicates location of region-of interest on the group average brain, left panel shows GD (red) and conGD (grey) data, right panel shows sibling (blue) and conSIB (grey) data. The bilateral caudate ROI: The GD group showed a significant difference between win and all miss outcomes $\left(x^{2}(1)=31.58, p<0.0001\right)$ but this difference was not modulated by group $\left(x^{2}(1)=0.0026, p=0.96\right)$. Siblings also showed a significant difference between win and all miss outcomes $\left(x^{2}(1)=\right.$ $18.12, p<.0001)$, but this difference was not modulated by group $\left(x^{2}(1)=2.01, p=0.16\right)$. The paracingulate cortex ROI: The GD group showed a significant difference between win and all miss outcomes $\left(x^{2}(1)=41.06, p<.001\right)$ but this difference was not modulated by group $\left(x^{2}(1)=\right.$ $0.00, p=.99)$. Siblings also showed a significant difference between win and all miss outcomes $\left(x^{2}(1)=27.56, p<0.0001\right)$, and this difference was modulated by group $\left(X^{2}(1)=4.05, p<0.05\right)$, with siblings showing reduced activity compared to the conSIBs group. The bilateral orbitofrontal cortex ROI: The GD group showed a significant difference between win and all miss outcomes $\left(x^{2}(1)=32.17, p<0.0001\right)$ but this difference was not modulated by group $\left(x^{2}(1)=1.02, p=0.33\right)$. Siblings also showed a significant difference between win and all miss outcomes $\left(x^{2}(1)=29.22, p<0.0001\right)$, but this difference was not modulated by group $\left(x^{2}(1)=1.22, p=0.27\right)$. GD gambling disorder, ROI region of interest

incentive salience (if GD severity should amplify gambling-related associations).

However, by using a cue-laden task based on slot machine gambling, our design may have been biased to detect incentive salience [61], and this may explain why we did not observe a between group difference that supported the reward deficiency hypothesis in our GD analysis. In addition, the lack of evidence for fMRI effects in the GD siblings tempers a vulnerability interpretation of our severity correlation. Similar negative correlations between gambling severity and striatal reward activation have been reported in the context of overall hypo-activation in GD $[25,27]$. Other studies have observed negative associations either 


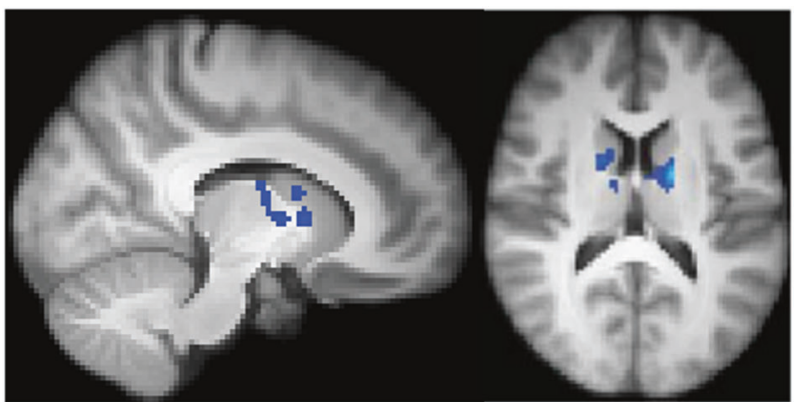

3.1 4

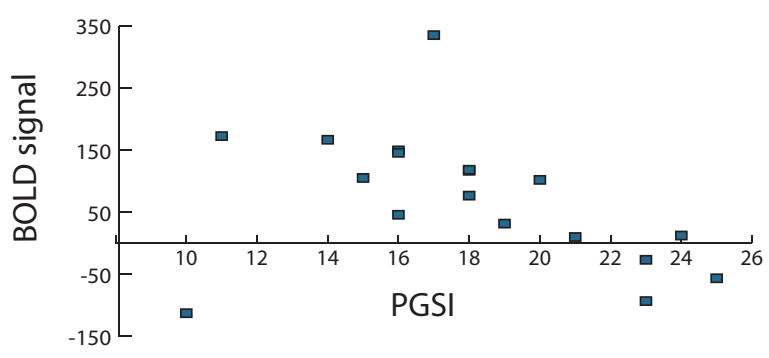

Fig. 4 Win $>$ all miss activity negatively correlates with PGSI score in the participants with Gambling Disorder. a A single cluster of activity peaks in white matter $[-16,-2,14]$, but extends to the caudate nuclei. b BOLD signal from this cluster extracted at the individual level. All images cluster corrected, $Z>3.1, p<0.05$ and presented using radiological convention. BOLD blood oxygen level dependent, PGSI problem gambling severity index

in the absence of an overall case-control difference (e.g. [62]), as seen here, or even in the context of group hyper-activation $[31,63]$.

With respect to our findings in the sibling group, it is those effects that are strongly and reliably associated with GD that we observed in the siblings. Increases in risky choice and moodrelated impulsivity (urgency) are consistently associated with GD $[14,18-21,64,65]$. Our findings that these signals overlap with the profile in GD mitigates against the possibility that differences in biological relatives could reflect resiliency against the disorder rather than risk [66]; this concern is arguably greater in adult samples who have passed the average age of onset [48]. However, caution is warranted against asserting genetic mediation of these findings, as biological siblings share both genes and environmental upbringing. Moreover, any vulnerability markers in firstdegree relatives are expected to be attenuated relative to the signal in actual cases, as genetics are only partially shared. Nevertheless, as a putative endophenotype, risky decision-making does meet criteria of heritability [67]. Specific gene variants affecting dopamine and serotonin have been implicated in GD [68-70] and variation in the cannabinoid receptor gene (CNR1) in a healthy sample affected Cambridge Gamble Task bet size [71].

It is notable that our evidence for endophenotypic signals in the GD siblings was observed on psychological measures, and our data do not support any changes on reward neuroimaging. It is often assumed that neuroimaging is the more sensitive procedure, although in other research looking at adolescent (age 14) markers of alcohol consumption, reward-related personality traits and genetic markers showed stronger signals than neuroimaging measures $[39,72]$. In a subset of the IMAGEN prospective study, a blunted ventral striatum response to reward anticipation at age 14 predicted drinking and smoking frequency at age $16[37,38]$. Cambridge Gamble Task risk-taking was available in the IMAGEN assessment, but was not a significant predictor of drug use. However, using a wheel of fortune task that isolates risky choice,
Morales et al. [73] saw that increased ventral striatal signal on high-risk decisions predicted earlier initiation of binge-drinking, as did a behavioural index of risky choice. The event-related structure may be critical here as both healthy adolescence and substance use disorders have been associated with reduced anticipatory signalling but enhanced activity to reward delivery [24, 74, 75]. These prior analyses, as well as the reward deficiency hypothesis of addiction vulnerability, centre on striatal signalling [76, 77]. By contrast, risk-taking and mood-related impulsivity may indicate a relatively greater role of orbital and lateral prefrontal pathophysiology $[65,78]$. Functional disruption in this circuitry is widely supported in GD $[25,30]$ but few studies speak directly to its role in vulnerability. Our findings are compatible with a recent structural MRI mega-analysis in GD showing a distinct profile of orbitofrontal morphology that is neurodevelopmental in origin [79].

We found no evidence of group differences in near-miss related activity, and the 'continue to play' ratings (delivered intermittently on $1 / 3$ trials) did not support any subjective effects of these outcomes. A previous study had reported increased striatal activation to near-misses in a GD group compared to healthy controls, with no corresponding difference in win-related activity [29]. This study used a shorter version of the slot machine task than prior research, which afforded reduced power to examine the near-miss outcomes, and drew from a treatment-seeking population, rather than through community advertisement.

As limitations, our sample of GD siblings was small due to challenges with recruitment, which is unsurprising for an illness that causes much strain within families [80]. A specific issue with the siblings approach in the present study was the unbalanced demographics, especially with respect to gender: the GD group were entirely male, whereas our siblings included both men and women. This in turn necessitated parallel comparisons using two subsets of a larger control group, with 9 overlapping male controls. The heritability of GD has been ascertained largely in male samples, with limited data in females [81]. There is also some evidence that the non-genetic environmental contribution to GD is mostly derived from non-shared (i.e. unique) environment [82] that may not be expressed in siblings. Although the siblings were screened carefully for mental illness, we have limited data on family history and so our study cannot ascertain the specificity of these markers to GD. Sensitivity analyses for assessing the impact of clinical variables (e.g. BDI depression) were limited by colinearity with gambling severity scores, and small sample size. We note that BDI score in individuals with GD has been shown to modulate the neural response to reward [62], and impaired decision-making has been observed in first-degree relatives of suicide completers [83] and obsessive compulsive disorder [84], which are conditions that share pathophysiological overlap with GD [79].

In conclusion, our investigation of unaffected siblings of people with GD identified impulsivity and risky choice as candidate endophenotypic markers of GD vulnerability. The observed sensitivity of self-reported and cognitive markers over reward neuroimaging helps to arbitrate between neuroscientific hypotheses of addiction vulnerability. Ongoing characterization of vulnerability markers may inform algorithms for ascertaining gambling risk and pave the way for targeted prevention strategies.

\section{ACKNOWLEDGEMENTS}

The authors wish to thank the study participants and the clinical team at Imanova, Centre for Imaging Sciences. The research was supported by the National Institute for Health Research (NIHR) Imperial Biomedical Research Centre. The views expressed are those of the author(s) and not necessarily those of the NHS, the NIHR, or the Department of Health. 


\section{FUNDING AND DISCLOSURE}

This study was funded by the Medical Research Council- MRC G1100554 (Clark). E.H.L.O. works as a postdoctoral fellow at the Centre for Gambling Research at UBC which is supported by funding from the Province of British Columbia and the British Columbia Lottery Corporation (BCLC), a Canadian Crown Corporation. She has received a speaker honorarium from the Massachusetts Council on Compulsive Gambling (U.S.A.) and accepted travel/accommodation for speaking engagements from the National Council for Responsible Gambling (U.S.A.), the International Multidisciplinary Symposium on Gambling Addiction (Switzerland) and the Responsible Gambling Council (Canada). She has not received any further direct or indirect payments from the gambling industry or groups substantially funded by gambling. ALH has received Honoraria paid into her Institutional funds for speaking and Chairing engagements from Lundbeck, Lundbeck Institute UK, Janssen-Cilag; received research grants or support from Lundbeck, GSK; consulted by but received no monies from Opiant and Lightlake. HBJ is Director of the NPGC London, Spokesperson on Behavioural Addictions for Royal College of Psychiatrists, and Board member of International Society of Addictions Medicine. LC is the Director of the Centre for Gambling Research at UBC, which is supported by funding from the Province of British Columbia and the British Columbia Lottery Corporation (BCLC), a Canadian Crown Corporation. LC receives funding from the Natural Sciences and Engineering Research Council (Canada). LC has received a speaker/travel honorarium from the National Association for Gambling Studies (Australia) and reviewing honoraria from the National Center for Responsible Gaming (US) and Gambling Research Exchange Ontario (Canada). He has not received any further direct or indirect payments from the gambling industry or groups substantially funded by gambling. He has provided paid consultancy to, and received royalties from, Cambridge Cognition Ltd. relating to neurocognitive testing. IM, REC, RSAF, ST have no sources of funding or potential conflict of interests to be declared.

\section{ADDITIONAL INFORMATION}

Supplementary Information accompanies this paper at (https://doi.org/10.1038/ s41386-019-0534-1)

Publisher's note Springer Nature remains neutral with regard to jurisdictional claims in published maps and institutional affiliations.

\section{REFERENCES}

1. Verdejo-García A, Lawrence AJ, Clark L. Impulsivity as a vulnerability marker for substance-use disorders: review of findings from high-risk research, problem gamblers and genetic association studies. Neurosci Biobehav Rev. 2008;32:777-810.

2. Bechara A. Risky business: emotion, decision-making, and addiction. J Gambl Stud. 2003;19:23-51.

3. Draganski B, Gaser C, Busch V, Schuierer G, Bogdahn U, May A. Neuroplasticity: changes in grey matter induced by training. Nature. 2004;427:311-2.

4. Gottesman II, Gould TD. The endophenotype concept in psychiatry: etymology and strategic intentions. Am J Psychiatry. 2003;160:636-45.

5. Ersche KD, Turton AJ, Pradhan S, Bullmore ET, Robbins TW. Drug addiction endophenotypes: impulsive versus sensation-seeking personality traits. Biol Psychiatry. 2010;68:770-3.

6. Robbins TW, Gillan CM, Smith DG, de Wit S, Ersche KD. Neurocognitive endophenotypes of impulsivity and compulsivity: towards dimensional psychiatry. Trends Cogn Sci. 2012;16:81-91.

7. Black DW, Monahan PO, Temkit M'hamed, Shaw M. A family study of pathological gambling. Psychiatry Res. 2006;141:295-303.

8. Walters GD. Behavior genetic research on gambling and problem gambling: a preliminary meta-analysis of available data. J Gambl Stud. 2001;17:255-71.

9. Mann K, Lemenager T, Zois E, Hoffmann S, Nakovics H, Beutel M, et al. Comorbidity, family history and personality traits in pathological gamblers compared with healthy controls. Eur Psychiatry. 2017;42:120-8.

10. Hodgins DC, Schopflocher DP, el-Guebaly N, Casey DM, Smith GJ, Williams RJ, et al. The association between childhood maltreatment and gambling problems in a community sample of adult men and women. Psychol Addict Behav. 2010;24:548-54

11. Slutske WS, Eisen S, True WR, Lyons MJ, Goldberg J, Tsuang M. Common genetic vulnerability for pathological gambling and alcohol dependence in men. Arch Gen Psychiatry. 2000;57:666-73.

12. Lobo DSS. Genetic aspects of gambling disorders: recent developments and future directions. Curr Neurol Neurosci Rep. 2016;3:58-66.

13. Kräplin A, Dshemuchadse M, Behrendt S, Scherbaum S, Goschke T, Bühringer G. Dysfunctional decision-making in pathological gambling: Pattern specificity and the role of impulsivity. Psychiatry Res. 2014;215:675-82.
14. Michalczuk R, Bowden-Jones H, Verdejo-Garcia A, Clark L. Impulsivity and cognitive distortions in pathological gamblers attending the UK National Problem Gambling Clinic: a preliminary report. Psychol Med. 2011;41:2625-35.

15. MacKillop J, Amlung MT, Few LR, Ray LA, Sweet LH, Munafò MR. Delayed reward discounting and addictive behavior: a meta-analysis. Psychopharmacol. 2011;216:305-21.

16. Chowdhury NS, Livesey EJ, Blaszczynski A, Harris JA. Pathological gambling and motor impulsivity: a systematic review with meta-analysis. J Gambl Stud. 2017;33:1213-39.

17. Kovács I, Richman MJ, Janka Z, Maraz A, Andó B. Decision making measured by the lowa Gambling Task in alcohol use disorder and gambling disorder: a systematic review and meta-analysis. Drug Alcohol Depend. 2017;181:152-61.

18. Wilson MJ, Vassileva J. Decision-making under risk, but not under ambiguity, predicts pathological gambling in discrete types of abstinent substance users. Front Psychiatry. 2018;9:239.

19. Zois $E$, Kortlang $N$, Vollstädt-Klein $S$, Lemènager $T$, Beutel $M$, Mann $K$, et al. Decision-making deficits in patients diagnosed with disordered gambling using the Cambridge Gambling task: the effects of substance use disorder comorbidity. Brain Behav. 2014;4:484-94.

20. Lawrence AJ, Luty J, Bogdan NA, Sahakian BJ, Clark L. Problem gamblers share deficits in impulsive decision-making with alcohol-dependent individuals. Addiction. 2009;104:1006-15.

21. Grant JE, Chamberlain SR, Schreiber LRN, Odlaug BL, Kim SW. Selective decisionmaking deficits in at-risk gamblers. Psychiatry Res. 2011;189:115-20.

22. Kreek MJ, Nielsen DA, Butelman ER, LaForge KS. Genetic influences on impulsivity, risk taking, stress responsivity and vulnerability to drug abuse and addiction. Nat Neurosci. 2005;8:1450-7.

23. Stevens L, Verdejo-García A, Goudriaan AE, Roeyers H, Dom G, Vanderplasschen W. Impulsivity as a vulnerability factor for poor addiction treatment outcomes: a review of neurocognitive findings among individuals with substance use disorders. J Subst Abus Treat. 2014;47:58-72.

24. Luijten M, Schellekens AF, Kühn S, Machielse MWJ, Sescousse G. Disruption of reward processing in addiction: an image-based meta-analysis of functional magnetic resonance imaging studies. JAMA Psychiatry. 2017;74:387-98.

25. Reuter J, Raedler T, Rose M, Hand I, Gläscher J, Büchel C. Pathological gambling is linked to reduced activation of the mesolimbic reward system. Nat Neurosci. 2005;8:147-8

26. De Ruiter MB, Veltman DJ, Goudriaan AE, Oosterlaan J, Sjoerds Z, Van Den Brink W. Response perseveration and ventral prefrontal sensitivity to reward and punishment in male problem gamblers and smokers. Neuropsychopharmacology. 2009;34:1027-38.

27. Balodis IM, Kober H, Worhunsky PD, Stevens MC, Pearlson GD, Potenza MN. Diminished frontostriatal activity during processing of monetary rewards and losses in pathological gambling. Biol Psychiatry. 2012;71:749-57.

28. Romanczuk-Seiferth N, Koehler S, Dreesen C, Wüstenberg T, Heinz A. Pathological gambling and alcohol dependence: neural disturbances in reward and loss avoidance processing. Addict Biol. 2015;20:557-69.

29. Sescousse G, Janssen LK, Hashemi MM, Timmer MHM, Geurts DEM, Ter Huurne $\mathrm{NP}$, et al. Amplified striatal responses to near-miss outcomes in pathological gamblers. Neuropsychopharmacology. 2016;41:2614-23.

30. Sescousse G, Barbalat G, Domenech P, Dreher J-C. Imbalance in the sensitivity to different types of rewards in pathological gambling. Brain. 2013;136:2527-38.

31. Miedl SF, Fehr T, Meyer G, Herrmann M. Neurobiological correlates of problem gambling in a quasi-realistic blackjack scenario as revealed by fMRI. Psychiatry Res. 2010;181:165-73.

32. Blum K, Gardner E, Oscar-Berman M, Gold M. 'Liking' and 'wanting' linked to Reward Deficiency Syndrome (RDS): hypothesizing differential responsivity in brain reward circuitry. Curr Pharm Des. 2012;18:113-8.

33. Gold MS, Badgaiyan RD, Blum K. A shared molecular and genetic basis for food and drug addiction: overcoming hypodopaminergic trait/state by incorporating dopamine agonistic therapy in psychiatry. Psychiatr Clin North Am. 2015;38:419-62.

34. Robinson TE, Berridge KC. Review. The incentive sensitization theory of addiction: some current issues. Philos Trans R Soc Lond B Biol Sci. 2008;363:3137-46.

35. Robinson $T E$, Berridge $K C$. The neural basis of drug craving: an incentivesensitization theory of addiction. Brain Res Brain Res Rev. 1993;18:247-91.

36. Limbrick-Oldfield EH, van Holst RJ, Clark L. Fronto-striatal dysregulation in drug addiction and pathological gambling: consistent inconsistencies? Neurolmage: Clin. 2013;2:385-93.

37. Büchel C, Peters J, Banaschewski T, Bokde ALW, Bromberg U, Conrod PJ, et al. Blunted ventral striatal responses to anticipated rewards foreshadow problematic drug use in novelty-seeking adolescents. Nat Commun. 2017;8:14140.

38. Whelan R, Watts R, Orr CA, Althoff RR, Artiges E, Banaschewski T, et al. Neuropsychosocial profiles of current and future adolescent alcohol misusers. Nature. 2014;512:185-9. 
39. Heinrich A, Müller KU, Banaschewski T, Barker GJ, Bokde ALW, Bromberg U, et al. Prediction of alcohol drinking in adolescents: Personality-traits, behavior, brain responses, and genetic variations in the context of reward sensitivity. Biol Psychol. 2016;118:79-87.

40. Ersche KD, Turton AJ, Chamberlain SR, Müller U, Bullmore ET, Robbins TW. Cognitive dysfunction and anxious-impulsive personality traits are endophenotypes for drug dependence. Am J Psychiatry. 2012;169:926-36.

41. Ferris J, Wynne $\mathrm{H}$. The Canadian problem gambling index. Final report. Ottawa: Canadian Centre on Substance Abuse; 2001.

42. Wechsler D. Wechsler abbreviated scale of intelligence: WASI. 1999.

43. Beck AT, Steer RA, Brown GK. Manual for Beck Depression Inventory-II. San Antonio, TX: Psychological Corporation; 1996.

44. Beck AT, Epstein N, Brown G, Steer RA. An inventory for measuring clinical anxiety: psychometric properties. J Consult Clin Psychol. 1988;56:893-831.

45. Bush K, Kivlahan DR, McDonell MB, Finn SD, Bradley KA. for the Ambulatory Care Quality Improvement Project. The AUDIT Alcohol Consumption Questions (AUDIT-C): An Effective Brief Screening Test for Problem Drinking. Arch Intern Med. 1998;158:1789.

46. Skinner HA. The drug abuse screening test. Addict Behav. 1982;7:363-71.

47. Heatherton TF, Kozlowski LT, Frecker RC, Fagerstrom K-O. The Fagerström test for nicotine dependence: a revision of the Fagerstrom Tolerance Questionnaire. Br J Addict. 1991:86:1119-27.

48. Kessler RC, Hwang I, LaBrie R, Petukhova M, Sampson NA, Winters KC, et al. DSMIV pathological gambling in the National Comorbidity Survey Replication. Psychol Med. 2008;38:1351-60.

49. Bernstein DP, Stein JA, Newcomb MD, Walker E, Pogge D, Ahluvalia $T$, et al. Development and validation of a brief screening version of the Childhood Trauma Questionnaire. Child Abus Negl. 2003;27:169-90.

50. Lesieur HR, Blume SB. The South Oaks Gambling Screen (SOGS): a new instrument for the identification of pathological gamblers. Am J Psychiatry. 1987;144:1184-8.

51. Cyders MA, Smith GT, Spillane NS, Fischer S, Annus AM, Peterson C. Integration of impulsivity and positive mood to predict risky behavior: development and validation of a measure of positive urgency. Psychol Assess. 2007;19:107-18.

52. Kirby KN, Petry NM, Bickel WK. Heroin addicts have higher discount rates for delayed rewards than non-drug-using controls. J Exp Psychol Gen. 1999;128:78-87.

53. Logan GD, Cowan WB, Davis KA. On the ability to inhibit simple and choice reaction time responses: a model and a method. J Exp Psychol Hum Percept Perform. 1984;10:276-91.

54. Rogers RD, Everitt BJ, Baldacchino A, Blackshaw AJ, Swainson R, Wynne K, et al. Dissociable deficits in the decision-making cognition of chronic amphetamine abusers, opiate abusers, patients with focal damage to prefrontal cortex, and tryptophan-depleted normal volunteers: evidence for monoaminergic mechanisms. Neuropsychopharmacology. 1999;20:322-39.

55. Chase HW, Clark L. Gambling severity predicts midbrain response to near-miss outcomes. J Neurosci. 2010;30:6180-7.

56. Clark L, Lawrence AJ, Astley-Jones F, Gray N. Gambling near-misses enhance motivation to gamble and recruit win-related brain circuitry. Neuron. 2009;61:481-90.

57. Clark L, Studer B, Bruss J, Tranel D, Bechara A. Damage to insula abolishes cognitive distortions during simulated gambling. Proc Natl Acad Sci USA. 2014;111:6098-103.

58. van Timmeren T, Zhutovsky P, van Holst RJ, Goudriaan AE. Connectivity networks in gambling disorder: a resting-state fMRI study. Int Gambl Stud. 2018;18:242-58.

59. Segarra N, Metastasio A, Ziauddeen $\mathrm{H}$, Spencer J, Reinders NR, Dudas RB, et al. Abnormal Frontostriatal Activity During Unexpected Reward Receipt in Depression and Schizophrenia: Relationship to Anhedonia. Neuropsychopharmacology. 2016:41:2001-10.

60. Clark L, Boileau I, Zack M. Neuroimaging of reward mechanisms in Gambling disorder: an integrative review. Mol Psychiatry. 2019;24:674-93.

61. Leyton M, Vezina P. Striatal ups and downs: their roles in vulnerability to addictions in humans. Neurosci Biobehav Rev. 2013;37:1999-2014.

62. Fauth-Bühler $M$, Zois $E$, Vollstädt-Klein $S$, Lemènager T, Beutel $M$, Mann K. Insula and striatum activity in effort-related monetary reward processing in gambling disorder: the role of depressive symptomatology. Neurolmage: Clin. 2014;6: 243-51.

63. van Holst RJ, Veltman DJ, Büchel C, Van Den Brink W, Goudriaan AE. Distorted expectancy coding in problem gambling: is the addictive in the anticipation? Biol Psychiatry. 2012;71:741-8.

64. Billieux J, Lagrange $G$, Van der Linden $M$, Lançon $C$, Adida $M$, Jeanningros R. Investigation of impulsivity in a sample of treatment-seeking pathological gamblers: a multidimensional perspective. Psychiatry Res. 2012;198:291-6.

65. Navas JF, Billieux J, Perandrés-Gómez A, López-Torrecillas F, Cándido A, Perales JC. Impulsivity traits and gambling cognitions associated with gambling preferences and clinical status. Int Gambl Stud. 2017;17:102-24.

66. Volkow ND, Wang G-J, Begleiter H, Porjesz B, Fowler JS, Telang F, et al. High levels of dopamine D2 receptors in unaffected members of alcoholic families. Arch Gen Psychiat. 2006;63:999.

67. Tuvblad C, Gao Y, Wang P, Raine A, Botwick T, Baker LA. The genetic and environmental etiology of decision-making: a longitudinal twin study. J Adolesc. 2013;36:245-55.

68. Wilson D, da Silva Lobo DS, Tavares H, Gentil V, Vallada H. Family-based association analysis of serotonin genes in pathological gambling disorder: evidence of vulnerability risk in the 5HT-2A receptor gene. J Mol Neurosci. 2013;49:550-3.

69. Lobo DSS, Aleksandrova L, Knight J, Casey DM, el-Guebaly N, Nobrega JN, et al. Addiction-related genes in gambling disorders: new insights from parallel human and pre-clinical models. Mol Psychiatry. 2015;20:1002-10.

70. Comings DE, Rosenthal RJ, Lesieur HR, Rugle LJ, Muhleman D, Chiu C, et al. A study of the dopamine D2 receptor gene in pathological gambling. Pharmacogenetics. 1996;6:223-34.

71. Qin H, Zeng J, Chen H, Deng L, Su L, Can Your DNA. Influence your bet-placing? the impact of cannabinoid receptor 1 gene on gambling tasks. Front Hum Neurosci 2018;12:458.

72. Nees F, Tzschoppe J, Patrick CJ, Vollstädt-Klein S, Steiner S, Poustka L, et al. Determinants of early alcohol use in healthy adolescents: the differential contribution of neuroimaging and psychological factors. Neuropsychopharmacology. 2012;37:986-95.

73. Morales AM, Jones SA, Ehlers A, Lavine JB, Nagel BJ. Ventral striatal response during decision making involving risk and reward is associated with future binge drinking in adolescents. Neuropsychopharmacology. 2018;43:1884-90.

74. Galvan A, Hare TA, Parra CE, Penn J, Voss H, Glover G, et al. Earlier development of the accumbens relative to orbitofrontal cortex might underlie risk-taking behavior in adolescents. J Neurosci. 2006;26:6885-92.

75. Hoogendam JM, Kahn RS, Hillegers MHJ, van Buuren M, Vink M. Different developmental trajectories for anticipation and receipt of reward during adolescence. Dev Cogn Neurosci. 2013;6:113-24.

76. Volkow ND, Wang GJ, Fowler JS, Logan J, Gatley SJ, Hitzemann R, et al. Decreased striatal dopaminergic responsiveness in detoxified cocaine-dependent subjects. Nature. 1997;386:830-3.

77. Hommer DW, Bjork JM, Gilman JM. Imaging brain response to reward in addictive disorders. Ann N Y Acad Sci. 2011;1216:50-61.

78. Clark L, Bechara A, Damasio H, Aitken MRF, Sahakian BJ, Robbins TW. Differential effects of insular and ventromedial prefrontal cortex lesions on risky decisionmaking. Brain. 2008;131:1311-22

79. Li Y, Wang Z, Boileau I, Dreher J-C, Gelskov S, Genauck A, et al. Altered orbitofrontal sulcogyral patterns in gambling disorder: a multicenter study. Transl Psychiatry. 2019;9:186.

80. Goodwin BC, Browne M, Rockloff M, Rose J. A typical problem gambler affects six others. Int Gambl Stud. 2017;17:276-89.

81. Slutske WS, Zhu G, Meier MH, Martin NG. Genetic and environmental influences on disordered gambling in men and women. Arch Gen Psychiatry. 2010;67:624-30.

82. Vitaro F, Hartl AC, Brendgen M, Laursen B, Dionne G, Boivin M. Genetic and environmental influences on gambling and substance use in early adolescence. Behav Genet. 2014;44:347-55.

83. Hoehne A, Richard-Devantoy S, Ding Y, Turecki G, Jollant F. First-degree relatives of suicide completers may have impaired decision-making but functional cognitive control. J Psychiatr Res. 2015;68:192-7.

84. Cavedini P, Zorzi C, Piccinni M, Cavallini MC, Bellodi L. Executive dysfunctions in obsessive-compulsive patients and unaffected relatives: searching for a new intermediate phenotype. Biol Psychiatry. 2010;67:1178-84. 